\title{
Dynamic Location Problem under Uncertainty with a regret based measure of robustness
}

\author{
Maria do Céu Marques* ${ }^{* \ddagger}$ and Joana Matos Dias ${ }^{\dagger \ddagger}$
}

\begin{abstract}
In this paper, we present a dynamic uncapacitated facility location problem that considers uncertainty in fixed and assignment costs as well as in the sets of potential facility locations and possible customers. Uncertainty is represented via a set of scenarios. Our aim is to minimize the expected total cost, explicitly considering regret. Regret is understood as a measure, for each scenario, of the loss incurred for not choosing that scenario's optimal solution if that scenario indeed occurred. We guarantee that the regret for each scenario is always upper bounded. We present a mixed integer programming model for the problem and we propose a solution approach based on Lagrangean relaxation integrating a local neighborhood search and a subgradient algorithm to update Lagrangean multipliers. The problem and the solutions obtained are first analyzed through the use of illustrative examples. Computational results over sets of randomly generated test problems are also provided.
\end{abstract}

keywords: dynamic location problems, uncertainty, scenarios, heuristics, Lagrangean relaxation

\section{Introduction}

The strategic nature of most location decision problems associated with the limited knowledge about problem parameters at the time of decision making, makes facility location problems under uncertainty an active area of research within the location research field. Models and solution methods that deal explicitly with uncertainties are even more complex than deterministic versions and with higher computational difficulties to achieve optimal or near-optimal solutions.

\footnotetext{
*Polytechnic Inst. of Coimbra, ISEC, DFM, Rua Pedro Nunes, 3030-199 Coimbra, Portugal. email: cmarques@isec.pt

${ }^{\dagger}$ Faculty of Economics, University of Coimbra, Av. Dias da Silva, 165, 3004-512 Coimbra, Portugal. email: joana@fe.uc.pt

${ }^{\ddagger}$ Institute for Systems Engineering and Computers at Coimbra (INESC Coimbra),Rua Antero de Quental, 199, 3000-033 Coimbra, Portugal
} 
During the last decades there has been considerable interest in location problems under uncertainty and a large volume of work is now available in specialized papers and monographs. Among the vast collection of works concerning facility location problems under uncertainty, we can find static (single-period) or dynamic (multi-period) approaches. Within the first class, we can include the following works, from earlier to most recent ones, reflecting the richness of the contributions to the field. We stress that this brief review has no pretensions of completeness. Louveaux (1986) presents a stochastic version of the classical uncapacitated facility location problem (UFLP) in which demands, variable production and transportation costs, and selling prices (incorporated in the model) can be random. The problem is formulated as a two-stage stochastic program, where the first-stage decisions are the location and the size (capacity) of the facilities to be established, and the second-stage decisions are the allocation of the available production to the most profitable demands. In that work also a stochastic version of the $p$-median, defined as a two-stage stochastic program, is presented, and relations between the stochastic versions of the $p$-median and the UFLP are discussed. Solution methods are later presented by Louveaux and Peeters (1992). The authors propose a heuristic dual-based procedure, inspired on the approach developed in (Bilde and Krarup, 1977; Erlenkotter, 1978) for the classical (static and deterministic) UFLP. Laporte et al. (1994) consider a capacitated facility location problem (CFLP) in which customer demands are stochastic. The problem consists of optimally determining the location and size of facilities given that future customer demand is uncertain. The objective function minimizes the difference between the sum of fixed facility costs and average cost of operating transportation services between facilities and customers (assignment costs), and the expected net revenue from supplying customers. The problem can also be viewed as a two-stage stochastic integer program. Current et al. (1997) address location problems in which the total number of facilities to be sited is uncertain. Two decision criteria are considered in $p$-median based formulations: the minimization of the maximum regret and the minimization of expected opportunity loss. Under the decision criteria, each problem locates an initial number of facilities when the total number is unknown. The approaches are illustrated with a sample problem. Serra and Marianov (1998) consider a $p$-median based model in which travel times between nodes and/or demand at nodes are uncertain, described by scenarios. Two $p$-median formulations are presented, the min-max and the regret approaches. The authors propose a heuristic method for both formulations, and a real application to the location of fire stations in Barcelona is presented. More recently, Berman and Drezner (2008) also consider the $p$-median problem when the total number of facilities to be sited in the future is uncertain. The problem seeks the location for $p$ facilities that minimize the expected weighted distance when up to $q$ new facilities are added to the system in the future. The probability of adding $0 \leq r \leq q$ new facili- 
ties (possible scenarios) is given. Heuristic algorithms are suggested to solve the problem (focusing in the case $q=1$ ). A similar integer programming model and a decomposition algorithm to solve it is presented by Sonmez and Lim (2012). As opposed to the previous work, in this paper the problem allows the closing of some of the facilities that were opened initially, due to future demand change, and considers also budget restrictions for the opening and closing of facilities. Ravi and Sinha (2004) propose a two-stage stochastic version of the UFLP and an 8 -approximation algorithm to solve it. Here, demand and fixed costs are both random, and facilities may be opened in either the first or second stage. A related two-stage stochastic program is proposed by Wang et al. (2011) in which service installation costs are also considered (services must be installed at the open facilities and each customer must be assigned to an open facility at which the service requested by the customer is installed). The authors propose a primal-dual approximation algorithm to solve the optimization problem. Lin (2009) proposes a stochastic version of the single-source capacitated facility location problem in which the demand is uncertain. The objective function is to minimize the total system costs including fixed facility costs and costs of servicing each demand point by its assigned facility. Simultaneously, recognizing that facilities should provide an adequate level of service, the model also incorporates facility service level requirements. These requirements are formulated as chance constraints, being the probability that each open facility can cope with the stochastic demand assigned.

Dynamic models are mainly concerned with planning the location and/or size of facilities over time such that the time dimension is explicitly represented through the use of time dependent decision variables. A dynamic location problem approach is usually necessary whenever the assignment costs change significantly during the planning horizon or there are significant costs for relocating facilities (Erlenkotter, 1981). This class of models are enriched with the answer to questions such as "when" to locate (Jacobsen, 1990). The reader is referred, for instance, to Ahmed and Garcia (2004), Romauch and Hartl (2005), Marques and Matos Dias (2013b), where explicitly dynamic location problems under uncertainty are proposed which can be viewed as two-stage stochastic approaches. Recent multi-stage stochastic approaches (in which uncertainty is resolved in more than one stage along the time horizon) are proposed by Hernández et al. (2012), Albareda-Sambola et al. (2013) and the references therein.

A related and important issue addressed in the literature is robustness, specially when faced with scenario-based models. A pioneer work about the use of the robustness concept in strategic management is presented in (Rosenhead et al., 1972). The criterion robustness is "a measure of the flexibility which an initial decision of a plan maintains for achieving near-optimal states in conditions of uncertainty". The proposed concept is developed through the case study of a factory location problem over time, and here the robustness concept refers to 
individual facilities, the ones that should be opened first, when considering a time horizon under uncertainty. The concept of robustness may have different meanings and interpretations though, being treated in the literature as a multifaceted issue (Roy, 2010). Dembo (1991) presents general coordinating models to achieve a reasonable (and feasible) solution to the stochastic programs, being as close as possible to the individual scenario solutions in a weighted norm sense. As emphasised by Dembo (1991), it might be very difficult to find a single solution defined as the best one considering all possible future scenarios. Under uncertainty, the concept of best solution strongly depends on the attitude towards risk of the Decision Maker. For instance, rather than the "optimal" solution for a specific scenario or even for the most likely scenario, a risk averse decision maker wants a robust decision, defined as the one that performs well across all scenarios and hedges against the worst of all possible scenarios (Kouvelis and $\mathrm{Yu}$, 1997). Different criteria can be used to select among robust solutions, such as min-max and min-max regret criteria (Aissi et al., 2009). A different robustness approach is given in (Mulvey et al., 1995). The authors consider both solution robust and model robust concepts: a solution is robust if it remains close to optimal for any scenario, and is model robust if it remains almost feasible for any scenario. As it is unlikely that a given solution will remain both feasible and optimal for all scenarios, the authors propose a multicriteria objective form that allows to measure the tradeoff between solution and model robustness. Snyder and Daskin (2006) introduces a new robustness measure for optimization under uncertainty, the stochastic $k$-robustness. In that work, the classical (static) $p-$ median and UFL problems with uncertain demands and transportation costs, described by probabilistic scenarios, are considered. The models minimize expected costs while making sure that the relative regret for each scenario is no greater than a pre-specified value $k$. The relative regret of a solution associated with a given scenario is calculated by the difference between the value of the solution under that scenario and the optimal value of the scenario divided by this latter value. The authors incorporate regret into the problems's formulations by considering constraints that guarantee that the relative regret associated with each solution, for each of the possible future scenarios, is upper bounded. They also propose a Lagrangian decomposition algorithm to solve the corresponding optimization problems. In a recent work (Lim and Sonmez, 2013) the same robustness measure is considered in a static facility $p$-median relocation problem. Several different robustness measures have been proposed in the literature that are able to generate solutions with different features to the problems. The reader is referred to (Dembo, 1991; Escudero et al., 1993; Ben-Tal and Nemirovski, 2000; Bertsimas and Sim, 2004; Snyder, 2006; Snyder and Daskin, 2006; Aissi et al., 2009; Bertsimas and Goyal, 2010; Roy, 2010) wherein the above and other robustness approaches are applied, discussed and compared, reflecting the importance of the subject. 
Inspired by these and other related works, in the present paper the dynamic uncapacitated facility location problem (DUFLP) under uncertainty described in (Marques and Matos Dias, 2013b) is revisited and a new version of the model is proposed as well as a solution approach to solve it. In this dynamic model, fixed and assignment costs are scenario dependent as well as the set of possible customers and the set of potential locations for facilities. The decisions to be made are where and when to locate the facilities, and how to assign the existing customers over the whole planning horizon and under each scenario, in order to minimize the expected total costs. At the same time, the relative regret for each scenario is constrained to be no greater than a pre-specified value $\alpha, \alpha \geq 0$. As far as the authors know, this measure is explicitly included in a dynamic location problem for the first time. The problem is formulated as an integer linear program and a solution approach based on Lagrangean relaxation is proposed.

The problem tackled in this paper can be applied in different real-world applications. It mainly describes any situation in which a company has to do the planning of strategic location investments over a given planning horizon with uncertainty. The main sources of uncertainty, here represented by possible future scenarios, come from the existence or lack of customers, as well as costs associated with the opening of facilities and satisfying the clients' demand. Costs for opening facilities can change due to the economic environment, behavior of the real estate market, changes in interest rates. Such costs can even hinder the opening of a facility. Assignment costs can change due to changes in road infrastructures, new roads can be built while others may become inaccessible, government policies, price of fuel, tolls, for instance. In such an uncertain environment, considering only those solutions that guarantee a bounded value for regret is indeed a possible way of making a robust decision. One possible application is in locating emergency facilities to deal with disaster management situations. Several different scenarios can be designed, considering the possibility of having several roads inaccessible, and having different levels of demand. The decision where to locate these services will consider a maximum regret value.

The remainder of this paper is organized as follows. In the following section, the notation used in this paper is introduced and the problem is described. In section 3 illustrative examples of the problem are discussed. In section 4 the solution approach is described. In section 5 computational experiments are described and results are discussed. Section 6 concludes this paper with some notes on future work.

\section{Problem description}

Consider a planning horizon represented by a discrete set of time periods $\mathcal{T}=$ $\{1, \ldots, t, \ldots, T\}$. The future will be one of a finite set of possibilities, represented by a discrete set of scenarios $\mathcal{S}=\{1, \ldots, s, \ldots, S\}$, where each scenario characterizes 
the value of all uncertain elements. Suppose that each $s \in \mathcal{S}$ will occur with probability $p^{s}$ such that $\sum_{s \in \mathcal{S}} p^{s}=1$.

Let the set of potential facility sites be denoted by $J=\{1, \ldots, j, \ldots, M\}$ and the set of possible customer locations (or demand points) by $I=\{1, \ldots, i, \ldots, N\}$. These sets include all the potential facility locations and all the potential customers for all possible scenarios, despite the fact that for each scenario in particular possibly only a subset of potential locations and a subset of customers is considered given the uncertainty associated with the existence of customers and the future existence of potential locations. Let us define $\delta_{i t}^{s}$ as equal to 1 if customer $i$ has a demand that has to be fulfilled during period $t$ for scenario $s$, and 0 otherwise. Then we have to guarantee that all customers such that $\delta_{i t}^{s}=1$ are assigned to an open facility, for all $(t, s) \in \mathcal{T} \times \mathcal{S}$.

In terms of costs, the model considers not only fixed costs (opening and operating), but also variable costs associated with the assignment of customers to the facilities. For $(j, t, s) \in J \times \mathcal{T} \times \mathcal{S}$, let $f_{j t}^{s}$ be the fixed cost of establishing (opening) facility $j$ at the beginning of period $t$ plus the operating and subsequent costs in period $t$, under scenario $s$; for $(i, j, t, s) \in I \times J \times \mathcal{T} \times \mathcal{S}, c_{i j t}^{s}$ represents the assignment cost of customer $i$ to facility $j$ in period $t$ and under scenario $s$. If it is not possible to open facility $j$ at the beginning of time period $t$, under scenario $s$, then the corresponding fixed cost will be considered equal to $+\infty$. Such a situation can only occur for $t>1$, given the possibility that any new service opens in that period. We assume that once a facility is opened, it stays open until the end of the planning horizon.

The decisions to be made are where and when to locate new facilities, and how to assign the existing customers over the whole planning horizon and under each scenario. Thus, we define the following binary decision variables: $x_{j t}$ equals 1 if facility $j$ is opened at the beginning of period $t$, and 0 otherwise; $y_{i j t}^{s}$ equals 1 if customer $i$ is assigned to facility $j$ in period $t$ and under scenario $s$, and 0 otherwise. Assignment decisions are considered to be taken a period at a time, and so they can be changed according to the scenario that comes true; on the other hand, location decisions are hard to revert, so we have to live with the decision taken whatever the scenario that came to occur. The objective will be the minimization of expected total cost, considering the uncertainty associated with the future.

For ease in the exposition, let us first formulate the problem for a given scenario $s \in \mathcal{S}$ as follows:

$$
\left(P_{s}\right) \quad \min \sum_{t \in \mathcal{T}} \sum_{j \in J} f_{j t}^{s} x_{j t}+\sum_{t \in \mathcal{T}} \sum_{i \in I} \sum_{j \in J} c_{i j t}^{s} y_{i j t}^{s}
$$

subject to

$$
\sum_{j \in J} y_{i j t}^{s}=\delta_{i t}^{s} \quad \forall i \in I, t \in \mathcal{T}
$$




$$
\begin{gathered}
\sum_{\tau=1}^{t} x_{j \tau}-y_{i j t}^{s} \geq 0 \quad \forall i \in I, j \in J, t \in \mathcal{T}, \\
\sum_{t \in \mathcal{T}} x_{j t} \leq 1 \quad \forall j \in J, \\
x_{j t} \in\{0,1\} \quad \forall j \in J, t \in \mathcal{T}, \\
y_{i j t}^{s} \in\{0,1\} \quad \forall i \in I, j \in J, t \in \mathcal{T} .
\end{gathered}
$$

The objective function (2.1) minimizes total costs (fixed plus variable costs). Constraints (2.2) require that in every time period, an existing customer is assigned to exactly one facility. Constraints (2.3) impose that an existing customer can only be assigned to open facilities. Constraints (2.4) ensure that each facility is opened at most once during the time horizon. Finally, (2.5)-(2.6) restrict the decision variables to be binary.

For a given $s \in \mathcal{S}$, let us represent the optimal objective function value of $\left(P_{s}\right)$ by $\xi_{s}^{*}$. If we could anticipate the scenario that will occur in the future, then the optimal solution would be easily calculated and it would be the optimal solution for the corresponding scenario (optimal solution of $\left(P_{s}\right)$ ). In the presence of uncertainty, a decision has to be made without prior knowledge of the scenario that will occur. This means that we can consider the existence of regret. In this context, regret can be understood as a measure of how much we will lose due to the fact that the optimal solution of the scenario $s$ that came to occur was not implemented. Our aim is to minimize the expected total cost guaranteeing that the regret for all scenarios is upper bounded: the solution to the problem is such that the objective function value under any scenario is at most $100 \alpha \%$ worse than the scenario's optimal solution. The problem can be formulated as follows:

$$
\begin{gathered}
\left(P_{\alpha}\right) \quad \min \sum_{t \in \mathcal{T}} \sum_{j \in J} \sum_{s \in \mathcal{S}} p^{s} f_{j t}^{s} x_{j t}+\sum_{s \in \mathcal{S}} \sum_{t \in \mathcal{T}} \sum_{i \in I} \sum_{j \in J} p^{s} c_{i j t}^{s} y_{i j t}^{s} \\
\text { subject to } \\
\sum_{t \in \mathcal{T}} \sum_{j \in J} f_{j t}^{s} x_{j t}+\sum_{t \in \mathcal{T}} \sum_{i \in I} \sum_{j \in J} c_{i j t}^{s} y_{i j t}^{s} \leq(1+\alpha) \xi_{s}^{*} \quad \forall s \in \mathcal{S}, \\
\sum_{j \in J} y_{i j t}^{s}=\delta_{i t}^{s} \quad \forall i \in I, t \in \mathcal{T}, s \in \mathcal{S}, \\
\sum_{\tau=1}^{t} x_{j \tau}-y_{i j t}^{s} \geq 0 \quad \forall i \in I, j \in J, t \in \mathcal{T}, s \in \mathcal{S}, \\
\sum_{t \in \mathcal{T}} x_{j t} \leq 1 \quad \forall j \in J,
\end{gathered}
$$




$$
\begin{gathered}
x_{j t} \in\{0,1\} \quad \forall j \in J, t \in \mathcal{T}, \\
y_{i j t}^{s} \in\{0,1\} \quad \forall i \in I, j \in J, t \in \mathcal{T}, s \in \mathcal{S} .
\end{gathered}
$$

The objective function (2.7) minimizes the expected total costs (fixed plus variable costs). Constraints (2.8) impose that relative regret for each scenario is no greater than $\alpha$. The remaining constraints are similar to the ones defined above for $\left(P_{s}\right)$. However, it should be pointed out that these constraints guarantee that a customer can be assigned to different facilities at different time periods and different scenarios, as the set of opened facilities is not scenario dependent.

This problem has $|J||\mathcal{T}|+|J||I||\mathcal{T}||\mathcal{S}|$ binary variables and $|\mathcal{S}|+|I||\mathcal{T}||\mathcal{S}|+$ $|J||I||\mathcal{T}||\mathcal{S}|+|J|$ constraints (not counting the zero-one constraints). Even for moderate dimensions of these sets, (2.7)-(2.13) becomes a quite large integer linear program. This problem can be seen as a generalization of the problem presented in (Marques and Matos Dias, 2013b).

\section{Expected total cost versus regret: illustrative exam- ples}

In this section, the effect of incorporating parameter $\alpha$ into the proposed dynamic location problem under uncertainty is illustrated. The tradeoff between the expected total cost and $\alpha$ will be also analysed. Three problem instances, randomly generated, will be considered. The problem $\left(P_{\alpha}\right)$ has been solved iteratively for several values of $\alpha$, and the best feasible solution found in each iteration was recorded. Initially, $\alpha$ was set to a large value and then it was reduced by 0.01 units at each iteration until no feasible solution could be found. Let us first consider an instance with 10 time periods, 20 potential facility sites, 100 possible customers and 5 scenarios. For this particular instance, it was possible to prove that $\left(P_{\alpha}\right)$ is infeasible for $\alpha<0.07$. The best expected total costs achieved for each $\alpha$ are plotted in Figure 1. We can see that the expected total cost has a non decreasing pattern as $\alpha$ decreases. In addition, the steep curve indicates that large reductions in regret are possible with small increases in expected total cost. These results are in accordance with similar results already observed in static models. Achieving a more robust solution can sometimes be accomplished by small changes in a given solution. This is depicted in Figure 2, where two situations are compared: considering a maximum relative regret of $19 \%$ and $7 \%$. For this particular example, we can see that small changes in location decisions can lead to more robust solutions.

Table 1 depicts the solutions in detail. We report the best objective function values found for some values of $\alpha$ as well as the corresponding location decisions. In column 'Increase' we report the increase (in percentage) of the best objective function values relative to the best one achieved with $\alpha=0.19$, given by the diference between the best objective function value for each $\alpha$ and the best one with 


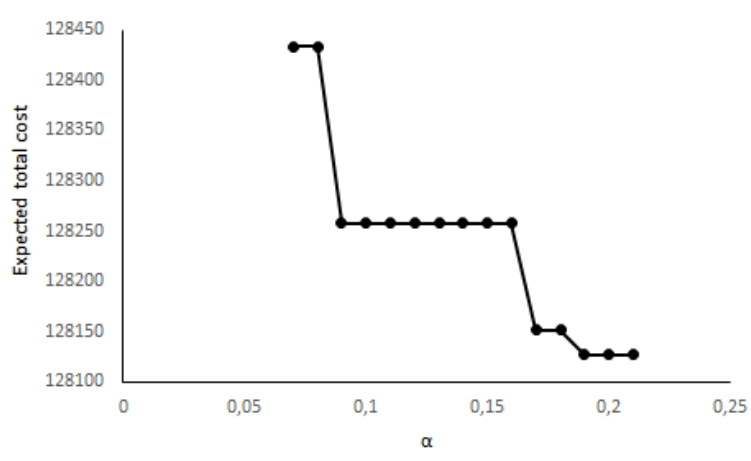

Figure 1: Expected total cost versus $\alpha$.

Table 1: Expected total cost versus $\alpha$.

\begin{tabular}{c|c|c|ccccc}
\hline$\alpha$ & Best Obj & Increase & Location Decisions & & & & \\
\hline & & & $\mathrm{t}=1$ & $\mathrm{t}=2$ & $\mathrm{t}=3$ & $\mathrm{t}=4$ & $\mathrm{t}=6$ \\
0.19 & 128127 & $0.0 \%$ & $9 ; 11 ; 13 ; 14 ; 17$ & $10 ; 18$ & 7 & 4 & 2 \\
0.17 & 128151,2 & $0.02 \%$ & $9 ; 11 ; 13 ; 14 ; 17$ & $10 ; 18$ & 7 & 4 & - \\
0.09 & 128257,8 & $0.09 \%$ & $6 ; 9 ; 11 ; 13 ; 14 ; 17$ & 18 & - & $4 ; 16$ & - \\
0.07 & 128433,4 & $0.24 \%$ & $9 ; 11 ; 13 ; 14 ; 17$ & 18 & - & $4 ; 16$ & 2 \\
\hline
\end{tabular}

$\alpha=0.19$ divided by this latter value. We can see that it is possible to decrease the relative regret from $19 \%$ to only $7 \%$ with a slightly increase of $0.24 \%$ in the expected objective function value (illustrated in Figure 2). Furthermore, we can gather adicional information about this particular problem, such as the discovery of a set of 'core' facilities, the ones that stay open for all values considered for parameter $\alpha$.

We will now consider two instances of the same size (example 2): 10 periods of time, 20 potential facility sites, 100 possible customers and 10 scenarios. The first instance proved to be infeasible for $\alpha<0.06$ and the second one for $\alpha<0.17$. The best solutions achieved for both problem instances, presented in Figure 3 and Table 2, show a behavior similar to the one observed in the previous instance. It is also possible to identify for both instances the corresponding set of core facilites.

The three instances used here for illustration purposes depict the general behavior observed in similar problems. It is also possible to see that each problem has its own features, and there can be huge variations in the obtained results (namely regarding the minimum relative regret value for which the problem is still feasible) even for problems of the same dimension. Looking at these examples, we can conclude that it is worthwhile to study the compromise that exists between expected total cost and maximum regret. The decision-maker will be able to 


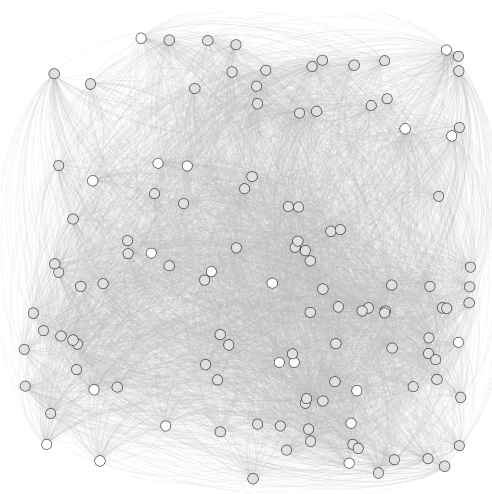

(a) Initial network

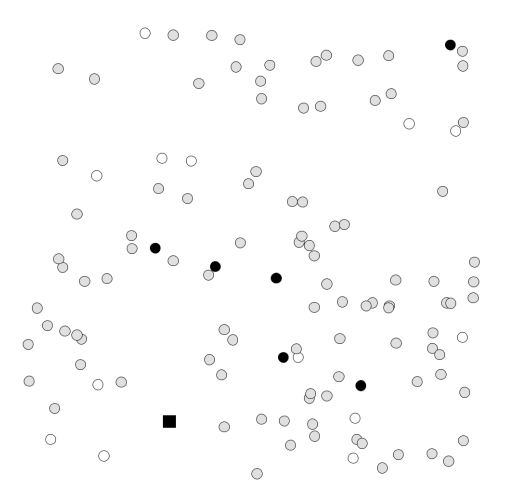

(c) $t=2$

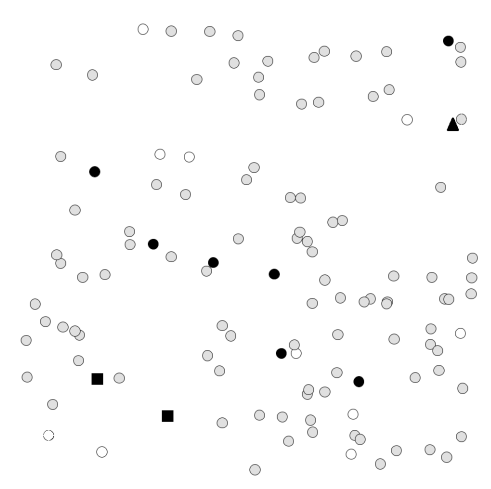

(e) $\mathrm{t}=4$

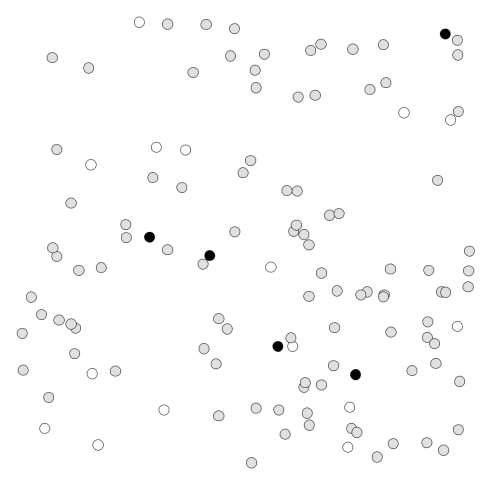

(b) $\mathrm{t}=1$

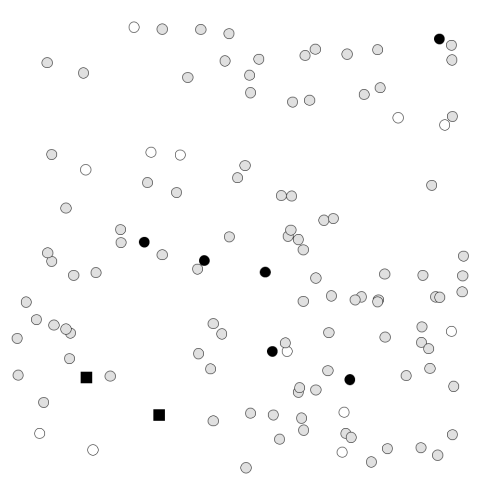

(d) $t=3$

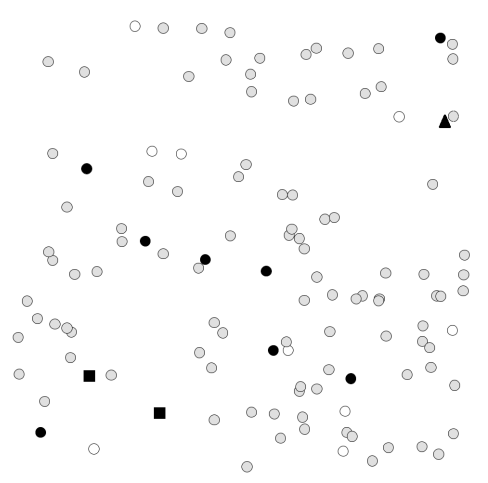

(f) $\mathrm{t}=6$

Figure 2: (a) Initial network. White nodes represent potential facility sites and gray nodes possible customers. (b) - (f) Networks with best location decisions. $(\bullet)$ represent facilities opened both for $\alpha=0.19$ and $\alpha=0.07$. ( $(\mathbf{\square})$ represent facilities opened only for $\alpha=0.19$. ( $\mathbf{\Lambda})$ represent facilities opened for $\alpha=0.07$. 


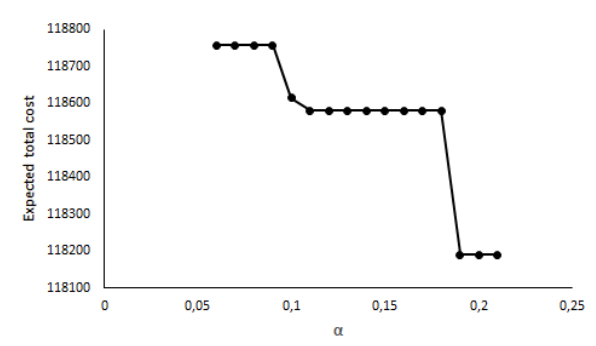

(a) Instance 1

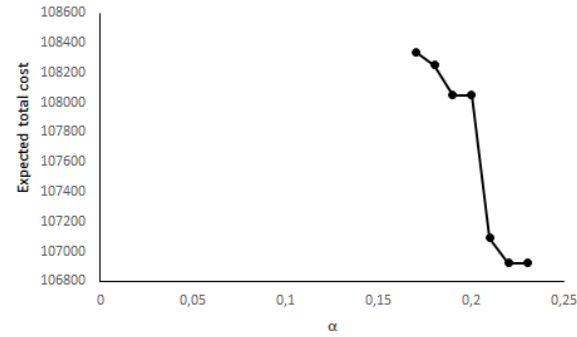

(b) Instance 2

Figure 3: Expected total cost versus $\alpha$ - example 2 .

Table 2: Expected total cost versus $\alpha$ - example 2.

\begin{tabular}{l|c|c|c|cccccc}
\hline & $\alpha$ & Best Obj & Increase & Loc Decisions & & & & \\
\hline \multirow{3}{*}{ Inst 1 } & & & & & $\mathrm{t}=1$ & $\mathrm{t}=2$ & $\mathrm{t}=3$ & $\mathrm{t}=4 \mathrm{t}=5 \mathrm{t}=6$ \\
& 0.19 & 118189.8 & $0.00 \%$ & $5 ; 7 ; 8 ; 14$ & $4 ; 12 ; 16$ & 18 & - & - & - \\
& 0.18 & 118580.0 & $0.33 \%$ & $5 ; 7 ; 8 ; 14$ & $12 ; 16$ & 18 & - & - & - \\
& 0.1 & 118614.8 & $0.36 \%$ & $5 ; 7 ; 8 ; 14 ; 20$ & $12 ; 16$ & 18 & - & - & - \\
& 0.06 & 118757.5 & $0.48 \%$ & $5 ; 7 ; 8 ; 14 ; 18$ & $12 ; 16$ & - & - & - & - \\
\hline Inst 2 & 0.22 & 106920.6 & $0.00 \%$ & $6 ; 7 ; 10$ & - & - & - & 5 & 17 \\
& 0.21 & 107088.5 & $0.16 \%$ & $6 ; 7 ; 10$ & - & 17 & - & 5 & - \\
& 0.2 & 108047.1 & $1.05 \%$ & $6 ; 10$ & - & 17 & - & 5 & - \\
& 0.18 & 108251.6 & $1.24 \%$ & $6 ; 10$ & - & 17 & 8 & 5 & - \\
& 0.17 & 108339.1 & $1.33 \%$ & $6 ; 10$ & - & $17 ; 20$ & - & 5 & - \\
\hline
\end{tabular}

make a more informed decision, choosing the solution that is most fitted to his attitude towards risk.

\section{Solution approach}

To be able to formulate and solve the problem, it is necessary to calculate the optimal solution $\xi_{s}^{*}$ for each scenario $s \in \mathcal{S}$. These $|\mathcal{S}|$ problems can be solved by the branch and bound procedure proposed in (Marques and Matos Dias, 2013a) or by a general solver (CPLEX, for instance). Assume then that $\xi_{s}^{*}$ is known and such that $\xi_{s}^{*}>0$, for all $s \in \mathcal{S}$.

Lagrangean relaxation is a well known technique that allows the calculation of lower bounds for integer programming problems (Reeves, 1993; Guignard, 2003). If constraints (2.8) are relaxed, the problem first introduced in (Marques and Matos Dias, 2013b) is obtained, allowing the use of an efficient primal-dual heuristic to calculate good quality solutions. This motivated the use of a Lagrangean relaxation and a subgradient algorithm, as described in the next subsection. 


\subsection{Lagrangean relaxation}

The Lagrangean relaxation of problem $\left(P_{\alpha}\right)$ with respect to the constraint set (2.8) can be defined through the introduction of the Lagrange multipliers $\lambda_{s} \geq$ $0, \forall s \in \mathcal{S}$. Each $\lambda_{s}$ is associated with the corresponding constraint and brought into the objective function, as follows:

$$
\begin{array}{r}
\left(L R P_{\alpha}\right) \quad \min \sum_{t \in \mathcal{T}} \sum_{j \in J} \sum_{s \in \mathcal{S}} p^{s} f_{j t}^{s} x_{j t}+\sum_{s \in \mathcal{S}} \sum_{t \in \mathcal{T}} \sum_{i \in I} \sum_{j \in J} p^{s} c_{i j t}^{s} y_{i j t}^{s}+ \\
\sum_{s \in \mathcal{S}} \lambda_{s}\left(\sum_{t \in \mathcal{T}} \sum_{j \in J} f_{j t}^{s} x_{j t}+\sum_{t \in \mathcal{T}} \sum_{i \in I} \sum_{j \in J} c_{i j t}^{s} y_{i j t}^{s}-(1+\alpha) \xi_{s}^{*}\right)
\end{array}
$$

subject to

$$
\text { (2.9)-(2.13). }
$$

The algorithm has been designed considering two well known results from Lagrangean Relaxation (e.g., Reeves (1993), Guignard (2003)) adapted for the present problem in the following proposition.

Proposition 4.1. The optimal solution of $\left(L R P_{\alpha}\right)$, for $\lambda_{s} \geq 0, \forall s \in \mathcal{S}$, gives a lower bound to the optimal solution of the original problem $\left(P_{\alpha}\right)$. In addition, a solution of $\left(L R P_{\alpha}\right)$ that satisfies also constraint set (2.8) provides an upper bound to the optimum of $\left(P_{\alpha}\right)$.

We have decided to use a very efficient primal-dual heuristic to solve problem $\left(L R P_{\alpha}\right)$. This heuristic has already been developed and applied to a problem that has the same structure of $\left(L R P_{\alpha}\right)$ (Marques and Matos Dias, 2013b). This dual-based heuristic is inspired on the classical approaches developed in (Bilde and Krarup, 1977; Erlenkotter, 1978) and (Van Roy and Erlenkotter, 1982). In order to apply the primal-dual heuristic to the present problem, it is necessary to formulate the dual problem of $\left(L R P_{\alpha}\right)$, in short $\left(\mathcal{D} L R P_{\alpha}\right)$, the so-called condensed dual problem, as well as the complementary slackness conditions between dual and primal problems. We present these formulations in appendix A where a summary of the heuristic is also given. The main idea of the approach is to obtain good solutions from the dual problem of the corresponding linear programming relaxation of the primal problem, more precisely from the so-called condensed dual problem. We stress that the heuristic is able to find admissible primal and dual solutions for feasible problems $\left(L R P_{\alpha}\right)$. Furthermore, the heuristic's procedures (dual ascent, primal and adjustment procedures) are designed to reduce progressively the duality gap between dual and primal objective function values. It is able to calculate very good quality solutions in reasonable computational times, even for large dimension instances. Even if the heuristic is unable to find 
the optimal solution of $\left(L R P_{\alpha}\right)$, it is still able to provide a good lower bound to the optimal objective function value of $\left(P_{\alpha}\right)$, in this case through the dual objective function value as stated in the next proposition.

Proposition 4.2. The best dual solution calculated by the primal-dual heuristic applied to $\left(L R P_{\alpha}\right)$ provides a lower bound to the optimal objective function value of $\left(P_{\alpha}\right)$.

Proof: Let us represent the optimum of $\left(P_{\alpha}\right)$ by $\mathcal{O p t}\left(P_{\alpha}\right)$ and the optimum of $\left(L R P_{\alpha}\right)$ by $\mathcal{O} p t\left(L R P_{\alpha}\right)$. In addition, let $\left(z_{P}, z_{D}\right)$ be the primal and dual solutions calculated by the primal-dual heuristic for $\left(L R P_{\alpha}\right)$ and its dual $\left(\mathcal{D} L R P_{\alpha}\right)$, respectively. If $z_{P}=z_{D}$, then $z_{P}=\mathcal{O} p t\left(L R P_{\alpha}\right)$ which provides a lower bound to $\mathcal{O} p t\left(P_{\alpha}\right)$ (proposition 4.1). If the heuristic's solutions are such that $z_{D}<z_{P}$, then, from duality theory, we know that $z_{D}<\mathcal{O} p t\left(L R P_{\alpha}\right) \leq \mathcal{O} p t\left(P_{\alpha}\right)$, so $z_{D}$ is a valid lower bound to $\mathcal{O} p t\left(P_{\alpha}\right)$.

Let us now turn to the generation of upper bounds. Taking into account (2.7) and the set of constraints (2.8), it is trivial to prove that the objective function value of $\left(P_{\alpha}\right)$ is bounded above by $\sum_{s} p^{s}(1+\alpha) \xi_{s}^{*}$. This value can then be considered as a first upper bound to the optimum of $\left(P_{\alpha}\right)$. Furthermore, if a lower bound calculated at any iteration is greater than this value, it means that $\left(P_{\alpha}\right)$ is infeasible.

The primal solution calculated by the heuristic can be admissible or not for $\left(P_{\alpha}\right)$. If it is admissible, then it represents an upper bound to the optimal solution of $\left(P_{\alpha}\right)$. After executing the primal-dual heuristic to $\left(L R P_{\alpha}\right)$, a local search procedure is performed. This local search procedure will explore the neighborhood of the current solution, trying to reach feasibility or trying to improve the objective function value (reaching better upper bounds). The neighborhood is considered to be the set of solutions that are equal to the current one with the exception of the opening time period of one facility. The local search procedure tries to change the time period when a given facility is opened, or tries to not open the facility at all. Whenever a better solution is found, it becomes the current solution and the local search continues until it is not possible to find better solutions in the neighborhood of the current solution.

A standard subgradient algorithm is used to update the Lagrange multipliers. Let us define subgradients $G_{s}$ for the relaxed constraints, evaluated at the current solution, by:

$$
G_{s}=\sum_{t \in \mathcal{T}} \sum_{j \in J} f_{j t}^{s} x_{j t}+\sum_{t \in \mathcal{T}} \sum_{i \in I} \sum_{j \in J} c_{i j t}^{s} y_{i j t}^{s}-(1+\alpha) \xi_{s}^{*}, \quad \forall s \in \mathcal{S} .
$$

In addition, let $\pi$ represent the step size for the Lagrange multipliers and $z$ the step size coefficients for the Lagrange multipliers.

Initially, in iteration $k=0, \lambda_{s}^{(k)}=0, \forall s \in \mathcal{S}$, 
Table 3: Dimension of the test problems.

\begin{tabular}{l|cccc|cc}
\hline Set & $|\mathcal{S}|$ & $|\mathcal{T}|$ & $|J|$ & $|I|$ & num var & num const \\
\hline I & 10 & 10 & 20 & 100 & 200200 & 210030 \\
II & 10 & 10 & 20 & 200 & 400200 & 420030 \\
III & 10 & 10 & 40 & 100 & 400400 & 410050 \\
IV & 10 & 20 & 20 & 100 & 400400 & 420030 \\
V & 20 & 10 & 20 & 100 & 400200 & 420040 \\
VI & 50 & 5 & 20 & 100 & 500100 & 525070 \\
\hline
\end{tabular}

and in iteration $k>0$,

$\lambda_{s}^{(k+1)}=\max \left\{0, \lambda_{s}^{(k)}+\pi G_{s}\right\}$, with $\pi=z \frac{U B^{(k)}-L B^{(k)}}{\sum_{s} G_{s}^{2}}$,

where $U B^{(k)}$ and $L B^{(k)}$ are the most recent upper and lower bounds achieved.

During the execution of the algorithm, the best upper and lower bounds achieved are updated and recorded, in order to calculate the solution gap, which is one of the established stopping criteria. The stopping criteria as other details of the algorithm will be discussed further in section 5 .

\section{Computational experiments}

In order to analyze the model and to assess the efficiency of the proposed algorithmic approach (both in terms of solution quality and computational time), several instances were randomly generated. Even though we are dealing with strategic decisions, where time usually is not determinant, faster algorithms permit the consideration of larger and diverse problems, enriching the decision making process. For this particular problem, being able to solve it for several different values of maximum regret will allow the decision-maker to get a better picture of the compromises that exist. However, it is desirable that this process takes place within a reasonable computational time. Six data sets were considered, with number of scenarios, number of time periods, number of possible facility locations and number of possible customers given in Table 3. The corresponding number of variables and constraints are also provided. For each one of these six sets, forty instances were randomly generated following the approach described in Appendix B. As we are in the presence of a dynamic problem under uncertainty, data must change simultaneously over time and among the different scenarios. All instances are available from the authors upon request.

We have considered $\alpha \in\{0.075,0.10,0.15,0.20\}$. The stopping criteria were established after some preliminary tests. The maximum computational time for the execution of the algorithm is two hours for problems with 20 and 50 scenarios and one hour for all other problems. In addition, we have also established as 
stopping criterium the quality of the best solution achieved by the algorithm, measured by the gap between the best known upper and lower bounds: $2 \%$ for the problems with 20 and 50 scenarios and $1.5 \%$ for all the others. We have also imposed a maximum number of iterations which could vary from 20 to 50 (largest instances). The computational results provided in this section were obtained considering a step size coefficient $z=1$ which gave the best results in general. Other initial values of $z$ as well as lowering $z$ after a few iterations of the algorithm were tested without significant improvements in results. The algorithm was coded in $\mathrm{C}$-language and the computational experiments were carried out on a AMD Turion(tm) X2 Dual-Core Mobile RM-70 processor at $2.00 \mathrm{GHz}$ with 3.00 GB of RAM.

Table 4 summarizes the computational results obtained. For each data set and for each $\alpha$, column 'feas/inf/ind' reports the number of instances for which a feasible solution was found by the algorithm, the number of instances identified as infeasible and also the number of instances for which the algorithm was unable to achieve a feasible solution (solution indeterminate). The statistics shown in the next columns refer only to the subsets of instances for which a feasible solution was found (feasible instances). For each $\alpha$ and for each feasible instance, the increase of the best objective function value relative to the best one achieved for $\alpha=0.2$ was calculated. Column 'increase' depicts the average increase (in percentage) obtained for each $\alpha$. The next columns report the minimum, average and maximum gap on the feasible instances, and the minimum, average and maximum time (in seconds) spent by the algorithm to solve each set of feasible instances (time results do not include the time required to read the problems' data, only the time to solve them). Gap is given, in percentage, by the difference between the best objective function value found by the algorithm and the best known lower bound on the optimal value divided by this best known lower bound. For each set, the last row shows the average results for gap and time.

We can see that the number of feasible instances decreases as $\alpha$ decreases in all sets, due to infeasibility of some instances or due to the algorithm being unable to achieve a feasible solution. The algorithm stopped with indeterminate solutions in only $7.6 \%$ of all 960 problems, due to the time limit established a priori, remaining the doubt about the feasibility of those instances. As expected, the objective function values increase as regret decreases. In terms of solution quality, the larger gaps were observed in sets V and VI, sets with larger number of scenarios, but the quality of the solutions is still very good. The worst gap equals $1.72 \%$ and was observed for instances with 50 scenarios. Apparently, the decrease of parameter $\alpha$ does not seem to cause a deterioration in the quality of the solutions in terms of gap, noticing however that the dimensions of the samples with problems for smaller values of $\alpha$ are very small. The computational time spent by the algorithm can vary a lot, even for problems within the same set (same size) and same $\alpha$. The higher execution times were observed in set III, 
with larger number of potential facility locations, and sets V and VI with larger number of scenarios.

We have solved the same sets of problems using an exact algorithm, CPLEX MIP optimizer, v12.4, with the same stopping criteria. The results are reported in Table 5. CPLEX stopped with indeterminate solutions in $10 \%$ of all 960 problems, due to lack of memory. Considering only set VI, CPLEX was unable to find a feasible solution in $19.4 \%$ of those 160 problems as Lagrangean relaxation approach stopped with indeterminate solutions in only $8 \%$. We noticed that within sets I to $\mathrm{V}$ the indeterminate instances of CPLEX were almost the same for which our algorithm was also unable to find a feasible solution, except 11 instances for which only our algorithm was able to find a feasible solution and 6 feasible instances only achieved by CPLEX. The results for these sets are very similar, reflecting that some instances are the hardest for both optimization algorithms. In terms of solution quality, CPLEX provides smaller average gaps than the Lagrangean relaxation approach, although less feasible instances were found by CPLEX, in particular in set VI with larger number of scenarios. In addition, CPLEX's maximum gap $1.97 \%$ is greater than the worst gap $1.72 \%$ achieved by the algorithm (achieved in sets V and VI, respectively, both for $\alpha=0.2$ ). In terms of computational time, CPLEX can also vary a lot. We can see that for all problems, the minimum computational time was obtained by the algorithm, in same cases clearly outperforming CPLEX. In terms of average computational times, CPLEX is better than the algorithm on sets III and VI, thought less feasible solutions were achieved by the solver.

In order to gather more information about the set of indeterminate instances, the computational time of one hour was increased to two hours in some of the sets. However, the algorithms were only able to find more infeasible instances, though very few.

In brief, the computational results show that the Lagragean relaxation approach is capable of finding very good quality solutions in reasonable computational times. It should be noted that CPLEX has better average gaps and computational times for some of the problems considered. However, for problems with larger number of scenarios the solver shows more difficulties to generate feasible solutions.

\section{Conclusions and future work}

In this paper we have described an uncapacitated discrete dynamic location problem that considers uncertainty in many of the problems' parameters through scenarios and explicitly considers regret. By the analysis of some illustrative examples, it was possible to obtain a deeper knowledge about the problem and its possible solutions: the possibility of achieving more robust solutions from small changes in a given and less robust solution, or the discovery of the core 
Table 4: Computational results.

\begin{tabular}{|c|c|c|c|c|c|c|c|c|c|}
\hline Set & $\alpha$ & feas/inf/ind & $\begin{array}{c}\text { increase } \\
(\%) \\
\text { mean }\end{array}$ & $\min$ & $\begin{array}{l}\text { gap } \\
(\%) \\
\text { mean }\end{array}$ & $\max$ & $\min$ & $\begin{array}{l}\text { time } \\
\text { (sec.) } \\
\text { mean }\end{array}$ & $\max$ \\
\hline \multirow[t]{5}{*}{ I } & 0.2 & $40 / 0 / 0$ & 0.00 & 0.00 & 0.23 & 1.05 & 9.95 & 119.13 & 873.9 \\
\hline & 0.15 & $39 / 0 / 1$ & 0.17 & 0.00 & 0.30 & 1.32 & 9.20 & 130.02 & 1621.2 \\
\hline & 0.1 & $32 / 8 / 0$ & 0.23 & 0.00 & 0.09 & 0.55 & 21.07 & 211.38 & 1567.9 \\
\hline & 0.075 & $14 / 26 / 0$ & 0.29 & 0.00 & 0.04 & 0.39 & 28.23 & 222.41 & 1031.7 \\
\hline & & & & 0.00 & 0.17 & 0.83 & 17.12 & 170.74 & 1273.7 \\
\hline \multirow[t]{5}{*}{ II } & 0.2 & $40 / 0 / 0$ & 0.00 & 0.00 & 0.09 & 0.51 & 1.2 & 327.2 & 1117.4 \\
\hline & 0.15 & $40 / 0 / 0$ & 0.19 & 0.00 & 0.25 & 1.14 & 1.3 & 352.7 & 1126.5 \\
\hline & 0.1 & $38 / 0 / 2$ & 0.25 & 0.00 & 0.19 & 0.99 & 1.3 & 449.2 & 1602.9 \\
\hline & 0.075 & $18 / 11 / 11$ & 0.26 & 0.00 & 0.12 & 0.90 & 30.2 & 585.9 & 3029.4 \\
\hline & & & & 0.00 & 0.17 & 0.89 & 8.47 & 428.77 & 1719.1 \\
\hline \multirow[t]{5}{*}{ III } & 0.2 & $40 / 0 / 0$ & 0.00 & 0.00 & 0.39 & 1.37 & 52.7 & 944.4 & 3609.0 \\
\hline & 0.15 & $40 / 0 / 0$ & 0.06 & 0.00 & 0.35 & 1.10 & 52.9 & 1008.3 & 3691.9 \\
\hline & 0.1 & $25 / 9 / 6$ & 0.14 & 0.00 & 0.25 & 0.83 & 98.2 & 789.1 & 3706.5 \\
\hline & 0.075 & $8 / 26 / 6$ & 0.18 & 0.00 & 0.17 & 0.64 & 97.9 & 807.6 & 3528.9 \\
\hline & & & & 0.00 & 0.29 & 0.99 & 75.42 & 887.33 & 3634.1 \\
\hline \multirow[t]{5}{*}{ IV } & 0.2 & $40 / 0 / 0$ & 0.00 & 0.00 & 0.29 & 1.46 & 5.5 & 303.2 & 2486.5 \\
\hline & 0.15 & $40 / 0 / 0$ & 0.24 & 0.00 & 0.45 & 1.46 & 5.5 & 367.2 & 1753.8 \\
\hline & 0.1 & $23 / 1 / 16$ & 0.28 & 0.00 & 0.33 & 1.33 & 5.5 & 586.4 & 3111.0 \\
\hline & 0.075 & $8 / 21 / 11$ & 0.29 & 0.00 & 0.12 & 0.68 & 5.6 & 289.5 & 742.2 \\
\hline & & & & 0.00 & 0.30 & 1.23 & 5.53 & 386.6 & 2023.4 \\
\hline \multirow[t]{5}{*}{ V } & 0.2 & $40 / 0 / 0$ & 0.00 & 0.00 & 0.31 & 1.59 & 88.4 & 480.3 & 2718.8 \\
\hline & 0.15 & $36 / 0 / 4$ & 0.37 & 0.00 & 0.32 & 1.51 & 88.6 & 630.7 & 1951.6 \\
\hline & 0.1 & $18 / 20 / 2$ & 0.44 & 0.00 & 0.28 & 1.05 & 127.0 & 769.9 & 3415.6 \\
\hline & 0.075 & $5 / 34 / 1$ & 0.48 & 0.00 & 0.14 & 0.52 & 128.6 & 505.4 & 1449.7 \\
\hline & & & & 0.00 & 0.26 & 1.17 & 108.2 & 596.6 & 2383.9 \\
\hline \multirow[t]{5}{*}{$\mathrm{VI}$} & 0.2 & $40 / 0 / 0$ & 0.00 & 0.00 & 0.24 & 1.72 & 59.4 & 929.8 & 3883.9 \\
\hline & 0.15 & $40 / 0 / 0$ & 0.03 & 0.00 & 0.19 & 1.24 & 57.6 & 1058.5 & 6631.6 \\
\hline & 0.1 & $33 / 3 / 4$ & 0.14 & 0.00 & 0.11 & 0.99 & 58.3 & 857.4 & 3124.8 \\
\hline & 0.075 & $17 / 14 / 9$ & 0.44 & 0.00 & 0.04 & 0.33 & 165.6 & 781.2 & 1608.7 \\
\hline & & & & 0.00 & 0.15 & 1.07 & 85.2 & 906.7 & 3812.3 \\
\hline
\end{tabular}


Table 5: Computational results using CPLEX.

\begin{tabular}{|l|l|c|ccc|ccc|}
\hline Set & & feas/inf/ind & \multicolumn{3}{|c}{ gap (\%) } & \multicolumn{3}{c|}{ time (sec.) } \\
& & & min & mean & max & min & mean & $\max$ \\
\hline I & 0.2 & $40 / 0 / 0$ & 0.00 & 0.15 & 1.26 & 54.40 & 130.48 & 1071.06 \\
& 0.15 & $39 / 0 / 1$ & 0.00 & 0.21 & 1.46 & 54.41 & 150.48 & 475.98 \\
& 0.1 & $32 / 8 / 0$ & 0.00 & 0.17 & 1.40 & 69.94 & 341.02 & 1711.57 \\
& 0.075 & $14 / 26 / 0$ & 0.00 & 0.01 & 0.13 & 61.04 & 177.86 & 488.88 \\
\hline & & & $\mathbf{0 . 0 0}$ & $\mathbf{0 . 1 3}$ & $\mathbf{1}$ & $\mathbf{5 9 . 9 5}$ & $\mathbf{1 9 9 . 9 6}$ & $\mathbf{9 3 6 . 8 7}$ \\
\hline II & 0.2 & $40 / 0 / 0$ & 0.00 & 0.04 & 0.91 & 160.4 & 232.8 & 404.9 \\
& 0.15 & $40 / 0 / 0$ & 0.00 & 0.05 & 0.91 & 159.9 & 292.7 & 947.4 \\
& 0.1 & $38 / 0 / 2$ & 0.00 & 0.10 & 0.77 & 158.9 & 567.3 & 3582.2 \\
& 0.075 & $18 / 11 / 11$ & 0.00 & 0.04 & 0.38 & 167.2 & 685.7 & 2206.7 \\
\hline & & & $\mathbf{0 . 0 0}$ & $\mathbf{0 . 0 6}$ & $\mathbf{0 . 7 4}$ & $\mathbf{1 6 1 . 6 1}$ & $\mathbf{4 4 4 . 6 2}$ & $\mathbf{1 7 8 5 . 2 8}$ \\
\hline III & 0.2 & $40 / 0 / 0$ & 0.00 & 0.29 & 1.37 & 138.3 & 404.5 & 1193.1 \\
& 0.15 & $38 / 0 / 2$ & 0.00 & 0.27 & 1.42 & 137.7 & 568.8 & 2218.1 \\
& 0.1 & $25 / 9 / 6$ & 0.00 & 0.17 & 1.08 & 144.6 & 877.0 & 3502.1 \\
& 0.075 & $8 / 26 / 6$ & 0.00 & 0.10 & 0.34 & 145.8 & 520.1 & 1596.8 \\
\hline & & & $\mathbf{0 . 0 0}$ & $\mathbf{0 . 2 1}$ & $\mathbf{1 . 0 5}$ & $\mathbf{1 4 1 . 5 9}$ & $\mathbf{5 9 2 . 6 2}$ & $\mathbf{2 1 2 7 . 5 1}$ \\
\hline IV & 0.2 & $37 / 0 / 3$ & 0.00 & 0.10 & 0.95 & 139.7 & 268.2 & 917.8 \\
& 0.15 & $36 / 0 / 4$ & 0.00 & 0.16 & 1.30 & 149.0 & 425.9 & 1999.4 \\
& 0.1 & $23 / 1 / 16$ & 0.00 & 0.15 & 0.65 & 161.4 & 793.4 & 3600.5 \\
& 0.075 & $12 / 21 / 7$ & 0.00 & 0.15 & 0.58 & 200.2 & 1116.5 & 3268.7 \\
\hline & & & $\mathbf{0 . 0 0}$ & $\mathbf{0 . 1 4}$ & $\mathbf{0 . 8 7}$ & $\mathbf{1 6 2 . 6 0}$ & $\mathbf{6 5 1 . 0 2}$ & $\mathbf{2 4 4 6 . 5 9}$ \\
\hline V & 0.2 & $40 / 0 / 0$ & 0.00 & 0.22 & 1.97 & 181.1 & 424.2 & 1861.5 \\
& 0.15 & $38 / 0 / 2$ & 0.00 & 0.32 & 1.51 & 201.7 & 934.7 & 4122.6 \\
& 0.1 & $16 / 20 / 4$ & 0.00 & 0.18 & 0.73 & 196.6 & 896.2 & 3567.0 \\
& 0.075 & $5 / 34 / 1$ & 0.00 & 0.00 & 0.00 & 198.8 & 409.3 & 784.4 \\
\hline & & & $\mathbf{0 . 0 0}$ & $\mathbf{0 . 1 8}$ & $\mathbf{1 . 0 5}$ & $\mathbf{1 9 4 . 6}$ & $\mathbf{6 6 6 . 1}$ & $\mathbf{2 5 8 3 . 8}$ \\
\hline VI & 0.2 & $37 / 0 / 3$ & 0.00 & 0.2 & 1.77 & 287.2 & 504.2 & 1106.1 \\
& 0.15 & $37 / 0 / 3$ & 0.00 & 0.11 & 1.72 & 293.9 & 520.7 & 1092.9 \\
& 0.1 & $25 / 3 / 12$ & 0.00 & 0.00 & 0.01 & 288.1 & 416.9 & 975.6 \\
& 0.075 & $13 / 14 / 13$ & 0.00 & 0.00 & 0.00 & 294.3 & 384.9 & 590.9 \\
\hline & & & $\mathbf{0 . 0 0}$ & $\mathbf{0 . 0 8}$ & $\mathbf{0 . 8 8}$ & $\mathbf{2 9 0 . 9}$ & $\mathbf{4 5 6 . 7}$ & $\mathbf{9 4 1 . 4}$ \\
\hline \hline
\end{tabular}


facilities, those that remain open even if the robustness parameter varies. An efficient Lagrangean relaxation technique was also developed to cope with this difficult problem, that is able to calculate very good quality solutions in reasonable computational times. In this model, an objective function that considers the minimization of the expected cost is being considered. We also intend to consider other objective functions, that can better represent the attitude towards risk of different decision-makers. In addition, capacity constraints, as well as the possibility of closing already opened facilities, should be considered to increase the range of applicability of the models.

\section{Acknowledgements}

This work has been partially supported by the Portuguese Foundation for Science and Technology under project grant PEst-OE/ EEI/UI308/2014. This work has been supported by project EMSURE (CENTRO 070224 FEDER 002004).

\section{References}

Ahmed, S. and Garcia, R. (2004). Dynamic capacity acquisition and assignment under uncertainty. Annals of Operations Research, 124:267-283.

Aissi, H., Bazgan, C., and Vanderpooten, D. (2009). Min-max and min-max regret versions of combinatorial optimization problems: a survey. European Journal of Operational Research, 197:427-438.

Albareda-Sambola, M., Alonso-Ayuso, A., Escudero, L. F., Fernandez, E., and Pizarro, C. (2013). Fix-and-relax-coordination for a multi-period locationallocation problem under uncertainty. Computers \& Operations Research, 40:2878-2892.

Ben-Tal, A. and Nemirovski, A. (2000). Robust solutions of linear programming problems contaminated with uncertain data. Mathematical Programming, 88:411-424.

Berman, O. and Drezner, Z. (2008). The p-median under uncertainty. European Journal of Operational Research, 189:19-30.

Bertsimas, D. and Goyal, V. (2010). On the power of robust solutions in two-stage stochastic and adaptive optimization problems. Mathematics of Operations Research, 35(2):284-305.

Bertsimas, D. and Sim, M. (2004). The price of robustness. Operations Research, $52(1): 35-53$. 
Bilde, O. and Krarup, J. (1977). Sharp lower bounds and efficient algorithms for the simple plant location problem. Annals of Discrete Mathematics, 1:79-97.

Current, J., Ratick, S., and ReVelle, C. (1997). Dynamic facility location when the total number of facilities is uncertain: A decision analysis approach. European Journal of Operational Research, 110:597-609.

Dembo, R. S. (1991). Scenario optimization. Annals of Operations Research, 30:63-80.

Erlenkotter, D. (1978). A dual-based procedure for uncapacitated facility location. Operations Research, 26(6):992-1009.

Erlenkotter, D. (1981). A comparative study of approaches to dynamic location problems. European Journal of Operational Research, 6(2):133-143.

Escudero, L. F., Kamesam, P. V., King, A. J., and Wets, R. J.-B. (1993). Production planning via scenario modelling. Annals of Operations Research, 43(6):311-335.

Guignard, M. (2003). Lagrangean relaxation. TOP, 11:151-228.

Hernández, P., Alonso-Ayuso, A., Bravo, F., Escudero, L. F., Guignard, M., Marianov, V., and Weintraub, A. (2012). A branch-and-cluster coordination scheme for selecting prison facility sites under uncertainty. Computers \& $O p$ erations Research, 39:2232-2241.

Jacobsen, S. K. (1990). Multiperiod capacitated location models. In Mirchandani, P. B. and Francis, R. L., editors, Discrete Location Theory, pages 173-208. John Wiley\&Sons.

Kouvelis, P. and Yu, G. (1997). Robust Discrete Optimization and its Applications. Kluwer Academic Publishers, Dordrecht.

Laporte, G., Louveaux, F. V., and Hamme, L. V. (1994). Exact solution to a location problem with stochastic demands. Transportation Science, 28(2):95103.

Lim, G. J. and Sonmez, A. D. (2013). $\gamma$-robust facility relocation problem. European Journal of Operational Research, 229(1):67-74.

Lin, C. K. Y. (2009). Stochastic single-source capacitated facility location model with service level requirements. International Journal of Production Economics, 117(2):439-451.

Louveaux, F. V. (1986). Discrete stochastic location models. Annals of Operations Research, 6:23-34. 
Louveaux, F. V. and Peeters, D. (1992). A dual-based procedure for stochastic facility location. Operations Research, 40(3):564-573.

Marques, M. C. and Matos Dias, J. (2013a). Dynamic location problem with uncertainty: a branch\&bound approach. In Oliveira, J. F. and Vaz, C. B., editors, Proceedings XVI Congresso da Associação Portuguesa de Investigação Operacional, pages 233-243. Instituto Politécnico de Bragança, Bragança, Portugal.

Marques, M. C. and Matos Dias, J. (2013b). Simple dynamic location problem with uncertainty: a primal-dual heuristic approach. Optimization, 62(10):13791397.

Mulvey, J. M., Vanderbei, R. J., and Zenios, S. A. (1995). Robust optimization of large-scale systems. Operations Research, 43(2):264-280.

Ravi, R. and Sinha, A. (2004). Hedging uncertainty: approximation algorithms for stochastic optimization problems. Lecture Notes in Computer Science, 3064:101-115.

Reeves, C. R. (1993). Modern Heuristic Techniques for Combinatorial Problems. Blackwell Scientific Publications, Oxford.

Romauch, M. and Hartl, R. F. (2005). Dynamic facility location with stochastic demands. Lecture Notes in Computer Science, 3777:180-189.

Rosenhead, J., Elton, M., and Gupta, S. K. (1972). Robustness and optimality as criteria for strategic decisions. Operational Research Quarterly, 23(4):413-431.

Roy, B. (2010). Robustness in operational research and decision aiding: A multifaceted issue. European Journal of Operational Research, 200:629-638.

Serra, D. and Marianov, V. (1998). The p-median problem in a changing network: the case of Barcelona. Location Science, 6:383-394.

Snyder, L. V. (2006). Facility location under uncertainty: a review. IIE Transactions, 38:537-554.

Snyder, L. V. and Daskin, M. S. (2006). Stochastic p-robust location problems. IIE Transactions, 38:971-985.

Sonmez, A. D. and Lim, G. J. (2012). A decomposition approach for facility location and relocation problem with uncertain number of future facilities. European Journal of Operational Research, 218:327-338.

Van Roy, T. J. and Erlenkotter, D. (1982). A dual-based procedure for dynamic facility location. Management Science, 28(10):1091-1105. 
Wang, X., Xu, D., and Zhao, X. (2011). A primal-dual approximation algorithm for stochastic facility location problem with service installation costs. Frontiers of Mathematics in China, 6(5):957-964. 


\section{A Summary of the primal-dual heuristic}

In order to apply the primal-dual heuristic to $\left(L R P_{\alpha}\right)$, the dual problem of $\left(L R P_{\alpha}\right)$, the condensed dual problem, and the complementary slackness conditions between dual and primal problems must be formulated.

In what follows, consider the objective function (4.1) of $\left(L R P_{\alpha}\right)$ rewritten as follows:

$$
\sum_{t \in \mathcal{T}} \sum_{j \in J} \sum_{s \in \mathcal{S}}\left(p^{s}+\lambda_{s}\right) f_{j t}^{s} x_{j t}+\sum_{s \in \mathcal{S}} \sum_{t \in \mathcal{T}} \sum_{i \in I} \sum_{j \in J}\left(p^{s}+\lambda_{s}\right) c_{i j t}^{s} y_{i j t}^{s}
$$

Notice that constant $-\sum_{s \in \mathcal{S}} \lambda_{s}(1+\alpha) \xi_{s}^{*}$ is not considered in (A.1), being added to the final objective function value. In addition, consider restrictions (2.11) rewritten as $\sum_{t \in \mathcal{T}}\left(-x_{j t}\right) \geq-1, \forall j \in J$.

Consider the linear programming relaxation of the primal problem $\left(L R P_{\alpha}\right)$ where restrictions (2.12) and (2.13) are replaced by nonnegativity constraints. Defining in (A.1) $\mathcal{F}_{j t}^{s}=\left(p^{s}+\lambda_{s}\right) f_{j t}^{s}$ and $\mathcal{C}_{i j t}^{s}=\left(p^{s}+\lambda_{s}\right) c_{i j t}^{s}$, and considering dual variables $v_{i t}^{s}, w_{i j t}^{s}$ and $u_{j}$ associated with the restrictions (2.9), (2.10) and (2.11), respectively, the dual problem of $\left(L R P_{\alpha}\right)$ is given by:

$\left(\mathcal{D} L R P_{\alpha}\right)$

$$
\begin{gathered}
\max \sum_{i \in I} \sum_{t \in \mathcal{T}} \sum_{s \in \mathcal{S}} \delta_{i t}^{s} v_{i t}^{s}-\sum_{j \in J} u_{j} \\
\text { subject to } \\
v_{i t}^{s}-w_{i j t}^{s} \leq \mathcal{C}_{i j t}^{s} \quad \forall i \in I, j \in J, t \in \mathcal{T}, s \in \mathcal{S}, \\
\sum_{i \in I} \sum_{s \in \mathcal{S}} \sum_{\tau=t}^{T} w_{i j \tau}^{s}-u_{j} \leq \sum_{s \in \mathcal{S}} \mathcal{F}_{j t}^{s} \quad \forall j \in J, t \in \mathcal{T}, \\
w_{i j t}^{s} \geq 0 \quad \forall i \in I, j \in J, t \in \mathcal{T}, s \in \mathcal{S}, \\
u_{j} \geq 0 \quad \forall j \in J .
\end{gathered}
$$

For feasible variables $v_{i t}^{s}$, by constraints (A.3) and (A.5), we may set

$$
w_{i j t}^{s}=\max \left\{0, v_{i t}^{s}-\mathcal{C}_{i j t}^{s}\right\} \quad \forall i, j, t, s,
$$

to obtain the condensed dual problem:

$$
\left(\mathcal{C D} L R P_{\alpha}\right) \quad \max \sum_{i \in I} \sum_{t \in \mathcal{T}} \sum_{s \in \mathcal{S}} \delta_{i t}^{s} v_{i t}^{s}-\sum_{j \in J} u_{j}
$$


subject to

$$
\begin{gathered}
\sum_{i \in I} \sum_{s \in \mathcal{S}} \sum_{\tau=t}^{T} \max \left\{0, v_{i \tau}^{s}-\mathcal{C}_{i j \tau}^{s}\right\}-u_{j} \leq \sum_{s \in \mathcal{S}} \mathcal{F}_{j t}^{s} \quad \forall j, t, \\
u_{j} \geq 0 \quad \forall j .
\end{gathered}
$$

The corresponding slack variables $\pi_{j t}$ for constraints (A.9) are given by:

$$
\pi_{j t}=\sum_{s \in \mathcal{S}} \mathcal{F}_{j t}^{s}-\sum_{i \in I} \sum_{s \in \mathcal{S}} \sum_{\tau=t}^{T} \max \left\{0, v_{i \tau}^{s}-\mathcal{C}_{i j \tau}^{s}\right\}+u_{j} \quad \forall j, t .
$$

Then, the complementary slackness conditions are:

$$
\begin{aligned}
\pi_{j t} x_{j t}=0 & \forall j, t, \\
v_{i t}^{s}\left(\sum_{j} y_{i j t}^{s}-\delta_{i t}^{s}\right) & =0 \quad \forall i, t, s, \\
w_{i j t}^{s}\left(\sum_{\tau=1}^{t} x_{j \tau}-y_{i j t}^{s}\right) & =0 \quad \forall i, j, t, s, \\
u_{j}\left(1-\sum_{t} x_{j t}\right) & =0 \quad \forall j, \\
y_{i j t}^{s}\left(v_{i t}^{s}-\mathcal{C}_{i j t}^{s}-w_{i j t}^{s}\right) & =0 \quad \forall i, j, t, s .
\end{aligned}
$$

The main steps of the heuristic are as follows:

1. Set $v_{i t}^{s}=\min _{j \in J}\left\{\mathcal{C}_{i j t}^{s}\right\}, \forall(i, t, s)$, and $u_{j}=0, \forall j$.

Set $I^{+}=\left\{(i, t, s) \in I \times \mathcal{T} \times \mathcal{S}: \delta_{i t}^{s}=1\right\}$.

2. Execute the dual ascent procedure.

3. Execute the primal procedure. If an optimal solution is found, then stop.

4. Execute the primal-dual adjustment procedure.

The heuristic stops when the optimal solution is found or when there are no primal or dual improvements after a given number of trials within the adjustment procedure. 
A formal and detailed description of this heuristic is given in (Marques and Matos Dias, 2013b). We can summarize it as follows. The ascent procedure starts with a dual feasible solution and tries to increase iteratively the values of dual variables associated with restrictions (2.9). This will lead to an increase of the dual objective function value and, simultaneously, to the decrease of some slacks' values. It stops when all dual variables are blocked from increasing by at least one slack, and thus no further improvements of the dual objective function value are possible. The output of this procedure is a dual feasible solution and an associated set of candidate facility locations defined by the slacks that are equal to zero. A corresponding candidate primal feasible solution will be constructed within the primal procedure. The facilities that are opened first, belonging to the set of candidate facilities that can be opened without violating complementary slackness conditions, are the ones that at a given time $t$ should be assigned to a given customer $i$ under some scenario $s$, the so-called essential facilities. Other facilities are only opened if strictly necessary, that is if exists a customer that cannot be assigned to an essential facility. Finally, as we are considering uncapacitated facilities, for each scenario $s$ and period $t$, each customer $i$ will be assigned to the facility operating in $t$ with the lowest assignment cost. If the dual and primal solutions satisfy all complementary slackness conditions, then the solutions are optimal and the heuristic stops. If not, the heuristic continues with adjustments in order to improve these solutions. Basically, it will try to enforce some complementary conditions that can still be violated. If this is the case, then at least one dual variable is decreased which causes the increase of at least two slacks, associated with distinct facilities, so some dual variables may be increased improving the dual objective value. As stated above, the heuristic stops when the optimal solution is found or when there are no primal or dual improvements after a given number of trials within the adjustment procedure. 
Table 6: Input values.

\begin{tabular}{ll}
\hline $\operatorname{MaxX}$ & 1000 \\
$\operatorname{Max} Y$ & 1000 \\
$p_{\text {arc }}$ & 0.75 \\
$d$ & 50 \\
$p_{\text {arcc }}$ & 0.80 \\
$p_{f}^{s}$ & 0.50 for $s=1$ and $0.7 \forall s \neq 1$ \\
$p_{c}^{s}$ & $0.60 \forall s$ \\
$p_{c}$ & 0.10 \\
$p_{a}^{s}$ & 0.70 \\
$p_{c f}^{s}$ & 0.90
\end{tabular}

\section{B Generation of test problems}

Below we provide the approach used in the generation of all test problems (in general). As far as scenario probabilities $\left(p^{s}\right)$ are concerned, these were randomly generated such that the sum of all probabilities is equal to 1 . Table 6 presents some input values that were considered and that must be known before the generation procedure.

For ease in the exposition, let us first consider the following additional notation:

$J_{t}^{s}$ : Set of potencial facility locations that can be selected (opened) at the beginning of time period $t \in \mathcal{T}$ for scenario $s \in \mathcal{S}$,

$I_{t}^{s}$ : Set of customer locations with demand during period $t \in \mathcal{T}$ for scenario $s \in \mathcal{S}$,

where $J_{t}^{s} \subseteq J$ and $I_{t}^{s} \subseteq I$.

1. Random generation of $(x, y)$-coordinates in a rectangular area of size $\operatorname{Max} X \times \operatorname{Max} Y$ corresponding to the location of $|J|+|I|$ nodes (potencial facility sites plus possible customer locations).

2. Random generation of arcs between the network nodes with probability $p_{\text {arc }}$; afterwards, if there isn't an arc between two nodes "close" (the Euclidean distance between them is less than $d$ ), an arc is created between them with probability $p_{\text {arcc }}>p_{\text {arc }}$.

3. For $s=1$ (basic scenario):

3.1 for $t=1$ : random generation of costs associated with arcs, according to a Uniform distribution $\mathcal{U}[l c, u c]$;

for each $t \geq 2$, each arc cost is equal to the cost in period $t-1$ plus a changing factor randomly generated. 
3.2 for each $t \geq 1$ :

i. calculation of the shortest path between each possible customer location and each potential facility location-assignment costsusing the Floyd-Warshall algorithm.

ii. random generation of set $J_{t}^{1}$, with $J_{1}^{1} \neq \emptyset$, and fixed costs: each location $j$ is included in $J_{t}^{1}$ with probability $p_{f}^{1}$;

- if $j \in J_{t}^{1}$, then the fixed cost at $j$ is randomly generated from a Uniform distribution $\mathcal{U}[l f, u f]$, and for each $\tau>t$ the fixed cost is increased by a changing factor randomly generated;

- if $j \notin J_{t}^{1}$, then the fixed cost at $j$ is set to $+\infty$.

iii. random generation of set $I_{t}^{1}$ : each customer $i$ is included in $I_{t}^{1}$ with probability $p_{c}^{1}$; in addition, for $t \geq 3$, if $i$ was included in $I_{t-2}^{1}$ and excluded from $I_{t-1}^{1}$, then $i$ is included in $I_{t}^{1}$ with probability $p_{c}<0.5$.

4. For $s \neq 1$ (other scenarios):

4.1 for $t=1$, consider the data generated for the basic scenario and $t=1$.

4.1 for each $t \geq 2$ :

i. each arc cost that was generated for time period $t$ of the basic scenario (basic cost) changes in time period $t$ of scenario $s$ with probability $p_{a}^{s}$; if a variation occurs, then the arc cost is equal to the basic cost plus a changing factor $\Theta_{a}$ randomly generated.

ii. calculation of the shortest path between each possible customer location and each potential facility location.

iii. random generation of set $J_{t}^{s}$ and fixed costs:

each location $j$ is included in $J_{t}^{s}$ with probability $p_{f}^{s}$;

- if $j \in J_{t}^{s} \cap J_{t}^{1}$, then the fixed cost at $j$ that was generated for time period $t$ of the basic scenario (basic cost) changes in time period $t$ of scenario $s$ with probability $p_{c f}^{s}$; if a variation occurs, then the fixed cost is equal to the basic cost plus a changing factor $\Theta_{f}$ randomly generated;

- if $j \in J_{t}^{s}$ but $j \notin J_{t}^{1}$, then the fixed cost at $j$ is randomly generated from a Uniform distribution $\mathcal{U}[l f, u f]$, and for each $\tau>t$ the fixed cost is increased by a changing factor randomly generated;

- if $j \notin J_{t}^{s}$, then fixed cost at $j$ is set to $+\infty$.

iv. random generation of set $I_{t}^{s}$ : the demand state of customer $i$ that was generated for time period $t$ of the basic scenario changes in time period $t$ of scenario $s$ with probability $p_{c}^{s}$. 